\title{
LABELED TREES AND RELATIONS ON GENERATING FUNCTIONS
}

\author{
M.P. DELEST, J.M. FEDOU \\ Bordeaux I University \\ LABR I + \\ Laboratory of Computer Science
}

\begin{abstract}
In this paper, we give a combinatorial interpretation for a property on generating functions gived by R. Stanley. Our proof is based upon the study of special kind of labeled trees and forests.
\end{abstract}

Résumé. Nous donnons ici une interprétation combinatoire d"une propriété des fonctions génératrices citée par R. Stanley. Notre preuve utilise une classe particulière d'arbres et de forêts étiquettếs.

\section{NTRODUCTION}

Trees are presents in many subjects of theorical computer science. There are the natural representation of a lot of objects such as programs, arithmetic expressions, words or algebraic expressions in language theory.

The structure of tree is very efficient in the study of complexity of algorithm because they constitute a dynamic data structure which allows to organize informations and to measure precisely the complexity of algorithm (see for example [4][6][11]). Trees are also present in other fields as shown in the paper of Viennot [14].

In some purely combinatorics subjects, they are the nice objects for understanding formulas. For example labeled trees, as defined in [2], are usefull in many subjects, see for instance Cori and Vauquelin [3] for the construction of a bijection between planar graphs and well labeled trees, or Moon [9] for identities on labelled forests. Moreover, the bijections are the basis of new theories in combinatorics (see for example [7], [13]) and trees are very studied in this context.

In this paper, we introduce a special kind of set of labeled trees, the k-shaped forests, in order to give a bijective proof and a generalization of a result about generating functions

\footnotetext{
+ Unité de Recherche Associée au Centre National de la Recherche Scientifique $n^{\circ} 726$.

* Post Mail : 351 Cours de la Libération, 33405 TALENCE Cedex, FRANCE.

* Electronic Mail: maylisegeocub.greco-prog.fr

* This work was supported by the "PRC de Mathématiques et Informatique".
} 
given by R. Stanley in [10] (see proposition 19 at the end of this paper). In fact, we study the generating functions

where

$$
F_{k}(X)=\sum_{n \geq 0} N_{k}(n) X^{n} \text {, }
$$

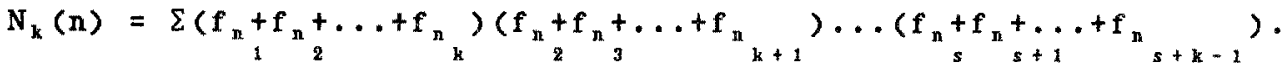

R. Stanley gives the result for $k=2$ and 3 , the proof only for $k=2$, saying that the formula for $k=3$ appears after an enormous amount of cancelation and tedious computation. Moreover he says that he does not known a simpler alternative method. We give it in paragraph 3 and in some way we generalize his result.

After some definitions and notations, in section 2 and 3 , we bring back the problem of the determination of $F_{k}(X)$ to the enumeration of $k$-shaped forests according to the number of sons of each vertex.

This problem of enumeration is then solved, in section 4, in the particular cases where $k=2,3$ and 4 . We solve a linear system of equations obtained by the mean of the k-shaped forests. The readers are refered to [4][5][14] for examples of similar methods.

\section{DEFINITIONS AND NOTATIONS}

A rooted tree is a connected graph G without cycle [1] with a distinguished vertex $r$ called root. If $(f, s)$ belongs to $G$, $f$ (resp. s) is called father (resp. son) of $s$ (resp. f). Here, we will consider trees which have sometimes a loop on the root. A set of trees is called a forest. A labeled tree is a tree with an integer, called label, associated to each vertex. The entering degree deg(i) of a vertex labelled is the number of sons of this vertex. The difference degree $\delta(i)$ of a vertex labeled i is the difference between the label of the father of this vertex and i. The difference degree of a loop is equal to 0 . It is easy to generalize these definitions to the forests. We note $\varepsilon$ the empty forest.

Let $K$ be an half $r$ ing and $Y$ be $\left\{y_{1}, y_{2}, \ldots, y_{k}\right\}$. We denote by K[Y] the $r$ ing of the formal power series over $Y$ with coefficients in $k$. Let $f(x)=\Sigma_{n}>0 f_{n} x^{n}$ and $g(x)=\Sigma_{n \geq 0} g_{n} x^{n}$ be two formal power series from $K[\{x\}]$, the product of Hadamard of $f$ and $g$ is defined by

$$
f * g(x)=\Sigma_{n>0} \quad f_{n} g_{n} x^{n}
$$

Generaly speaking, we will denote by $(* f)^{p}$ the product of Hadamard $p$ times of the serie $f$ that is $(* f)^{p}=\Sigma_{n>0} f_{n}^{p} x^{n}$. By convention, we note

$$
A=(* f)^{0}=\Sigma_{n>1} x^{n}=x /(1-x) .
$$

We call parameter an application from $\mathbb{N}$ into $N$. The two degrees deg and $\delta$ are parameters. 


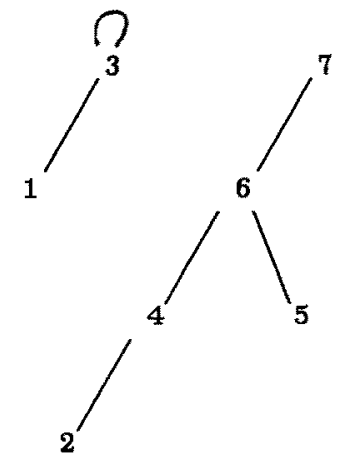

8

Figure 1. A forest of $\mathscr{S}_{3,6}$.

\section{K-SHAPED FORESTS}

We introduce a special kind of labeled forest, in order to "explain" products such as $\left(y_{1}+\ldots+y_{k}\right) \ldots\left(y_{s}+\ldots+y_{s+k-1}\right)$.

Definition 1. Let be a forest having t vertices labeled from 1 to $t$. The forest is $\mathrm{k}$-labeled when, for every 1 in [1.t]

$$
0 \leqslant \delta(i)<k \text {. }
$$

Definition 2. A k-labeled forest 4 is said k-shaped if 4 has $\mathrm{s}+\mathrm{k}-1$ vertices with $s \geq 0$ such that

i) every root in $A$ labeled with 1 in [1..s] has a loop,

ii) the k-1 last vertices labeled from $s+1$ to $s+k-1$ are roots without loop.

\section{Notations.}

$\mathscr{P}_{h}$, is the set of k-shaped forests having $s+k-1$ vertices.

$\mathscr{S}_{\mathrm{h}, 0}$ is the unique forest made with k-1 isolated vertices, we denote it by e.

$\mathscr{I}_{k}$ is the set of k-shaped forests.

See for example the figure 1 , the forest $A$ belongs to $\mathscr{S}_{3,6}$.

Remark 3. Let $\mathscr{A}$ be in $\mathscr{S}_{k, s}$, we obtain k forests in $\mathscr{F}_{k, s+i}$ by the mean of the following algorithm:

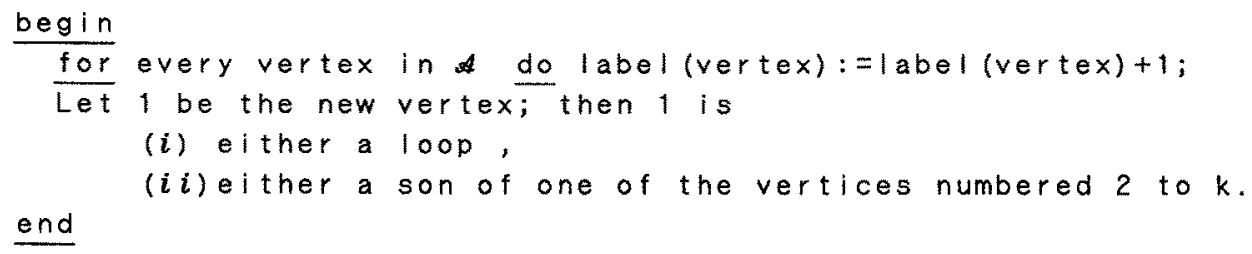

Thus, the number of k-shaped forests having $n+k-1$ vertices is $k^{n}$. 

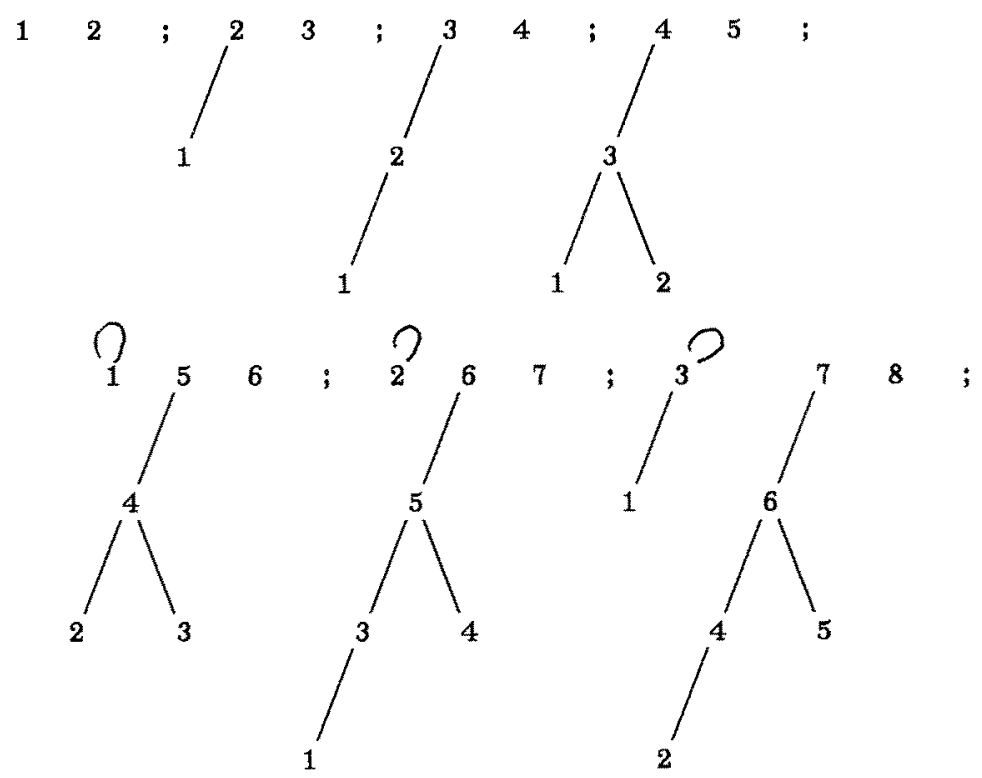

Figure 2. An example of the algorithm of remark 3

The figure 2 shows the construction of the forest of figure 1 using this algorithm. In this example, we have $k=3$.

Remark 4. Let $P$ be the product

$$
\left(y_{1}+y_{2}+y_{3}\right)\left(y_{2}+y_{3}+y_{4}\right)\left(y_{3}+y_{4}+y_{5}\right)\left(y_{4}+y_{5}+y_{6}\right)\left(y_{5}+y_{6}+y_{7}\right)\left(y_{6}+y_{7}+y_{8}\right) .
$$

In some way, we can say that the forest of figure 1 "represents" the term $y_{3}{ }^{2} y_{4} y_{6}{ }^{2} y_{7}$ in the development of $P$. In fact, on this forest every relation " $j$ is father of $i$ " means "we choose $y_{j}$ in the $i^{\text {th }}$ factor $\left(y_{i}+y_{i+1}+y_{i+2}\right)$ ".

Let p be a parameter.

Definition 5. The weight according to $p$ of a forest from $\mathscr{S}_{\mathrm{k}, \mathrm{s}}$ is the monomial defined by

$$
\pi_{p}(d)\left(y_{1}, \ldots, y_{s+k-1}\right)=\prod_{1<i<s+k-1} y_{i}^{p(i)} \text {. }
$$

For example, if $A$ is the forest of figure 1 , the weights according to the parameters entering degree and difference degree are repectively

and

$$
\pi_{d e g}(A)=y_{3}^{2} y_{4} y_{6}^{2} y_{7},
$$

$$
\pi_{\delta}(A)=\mathrm{y}_{1}^{2} \mathrm{y}_{2}^{2} \mathrm{y}_{4}^{2} \mathrm{y}_{5} \mathrm{y}_{6} \text {. }
$$


Notation. For every forest $A$ from $\mathscr{S}_{k, s}$, let $\varphi$ be the map from $\mathscr{S}_{k}$ into $\mathbb{Z}[Y]$ defined by

$$
\varphi(A)=\prod_{i=1}^{s+k-1} y_{d \in g(i)} .
$$

Define the formal power serie $\Phi\left(\mathscr{P}_{k}\right)$ of $\mathbb{Z}[Y]$ by

$$
\Phi\left(\mathscr{P}_{\mathrm{k}}\right)=\sum_{\mathscr{A} \in \mathscr{S}_{\mathrm{k}}} \varphi(\mathscr{A}) .
$$

$\Phi\left(\mathscr{\varphi}_{\mathrm{h}}\right)$ is the generating function of the k-shaped forest accurding to the number of sons of the vertices that is

$$
\Phi\left(\varphi_{k}\right)=\sum_{i_{0}, \ldots, i_{k-1}}^{n_{i} \ldots i_{k} Y_{0}^{i}{ }^{0} \ldots Y_{k}^{i}{ }^{k},}
$$

where $n_{i} \ldots i_{k}$ is the number of k-shaped forests such that, for each $j$ in $[0 . . k]$, there is i vertices which have $j$ sons.

Definition 6. Let $f(x)=\Sigma_{n>1} f_{n} x^{n}$ be a formal power serie of $\mathrm{K}[\mathrm{x}]$ and ${ }_{\text {be }}$ an element of $\mathscr{S}_{\mathrm{k}, \mathrm{s}}$. We denote by $\Delta_{\mathrm{f}}$ the map from $\mathscr{S}_{\mathrm{k}}$ into $\mathbb{Z}[x]$ defined by

$$
\Delta_{P}(*)=\prod_{1 \leqslant i \leqslant s+k-1}(* f)^{d \cdot k(i)} .
$$

Property 7. $\Delta_{\mathrm{f}}$ and $\Pi_{\mathrm{d} e \mathrm{~g}}$ are morphisms over the forests.

We obtain $\Delta_{\mathrm{f}}(A)$ substituting formally to each $\mathrm{y}_{\mathrm{i}}$ the value (*f) in $\Pi_{d e g}(A)$ or the value $(* f)^{i}$ in $\varphi(A)$. Moreover, let $\left\{\mathrm{u}, \mathbb{A}^{\prime}\right\}$ be a forest where $u$ is a tree and a forest, applying the previous definitions to the labeled trees gives the following equalities which prove the property 7

and

$$
\Delta_{\mathrm{f}}\left(\left\{\mathrm{u}, \Delta^{\prime}\right\}\right)=\Delta_{\mathrm{P}}(\mathrm{u}) \Delta_{\mathrm{P}}\left(A^{\prime}\right)
$$

$$
\Pi_{d \in g}(\{u, A\})=\Pi_{d \in g}(u) \Pi_{d \in g}(A) \text {. }
$$

Examples: In $\mathscr{S}_{\mathrm{k}}$, we have $\Delta_{\mathrm{g}}(\varepsilon)=\mathrm{A}^{\mathrm{k}-1}$. If $A$ is the forest of figure 1 , we have $\Delta_{f}(A)=A^{4} f^{2}(* f)^{2}$ and $\varphi(A)=Y_{0}{ }^{4} Y_{1}{ }^{2} Y_{2}{ }^{2}$. 
Definition 8 . Let $A_{1}$ and $A_{2}$ be two forests from $\mathscr{S}_{\mathrm{k}, \mathrm{s}}$ " The forests $A_{1}$ and $A_{2}$ are equivalents when, if

$$
\Pi_{d \in g}\left(A_{1}\right)=y_{1}^{n}{ }^{1} \ldots y^{n}{ }^{n+k+k}-1
$$

then there exists a permutation of $[1 \ldots s+\mathrm{k}-1]$ such that

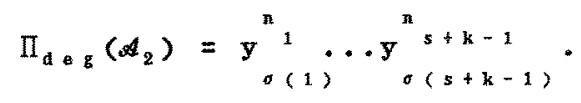

The property 7 gives immediatly the following proposition.

Proposition 9. Let $A_{1}$ and $A_{2}$ be two equivalent forests from $\mathscr{S}_{\mathrm{K}, \mathrm{s}}$. We have the following properties

$$
\begin{aligned}
& \text { (i) } \varphi\left(A_{1}\right)=\varphi\left(A_{2}\right), \\
& \text { (i i) if } \mathrm{f} \in \mathrm{K}[\mathrm{x}], \Delta_{\mathrm{p}}\left(A_{1}\right)=\Delta_{\mathrm{P}}\left(A_{2}\right) .
\end{aligned}
$$

\section{INTERPRETATION OF $F_{k}$ IN TERM OF $k$-SHAPED FORESTS}

In this paragraph, we give an expression for $F_{k}(X)$ using $\mathscr{P}_{\text {In }}$ that is

Theorem 10. The function $\mathrm{F}_{\mathrm{k}}(\mathrm{x})$ is deduced from the enumerating function $\Phi\left(\mathscr{S}_{\mathrm{k}}\right)$ of the k-shaped farests substituting formaly each $\mathrm{Y}_{\mathrm{i}}$ by $(* \mathbf{f})^{i}$.

First, we give some precision about $F_{k}$. Define, for every integers $\mathrm{k}, \mathrm{s}$ and $\mathrm{n}$

$N_{k, s}(n)=\sum\left(f_{n}+f_{n}+\ldots+f_{n}\right)\left(f_{n}+f_{n}+\ldots+f_{n}\right) \ldots\left(f_{n}+f_{n}+\ldots+f_{n}\right) \ldots$ where the sum is taken over all ordered partitions of $n=n_{1}+n_{2}+\ldots n_{s+k-1}$ with $n_{i} \geqslant 1$ and

$$
F_{k, s}(x)=\Sigma_{n \geq 1} N_{n, s}(n) x^{n} \text {. }
$$

Note that we have

and also

$$
N_{k}(n)=\Sigma_{s}>0 N_{k, s}(n), \quad F_{k}(x)=\Sigma_{s}>0 F_{k, s},
$$

$$
F_{k, s}(x)=\sum P_{k, s}\left(f_{p_{1}}, \ldots, f_{p} f_{s+k-1}\right) x^{n} \text {, }
$$

where the sum is over all ordered partitions of $n$ with $n_{i} \geqslant 1$.

Let $P_{k, s}\left(y_{1}, \ldots, y_{s+k-1}\right)$ be the polynomial of $\mathbb{Z}[Y]$ equal to 1 if $s=0$ and otherwise given by

$$
P_{k, s}\left(y_{1}, \ldots, y_{s+k-1}\right)=\left(y_{1}+\ldots y_{k}\right)\left(y_{2}+\ldots+y_{k+1}\right) \ldots\left(y_{s}+\ldots+y_{s+k-1}\right)
$$


In order to prove the theorem 10 we prove the following

Lemma 11.

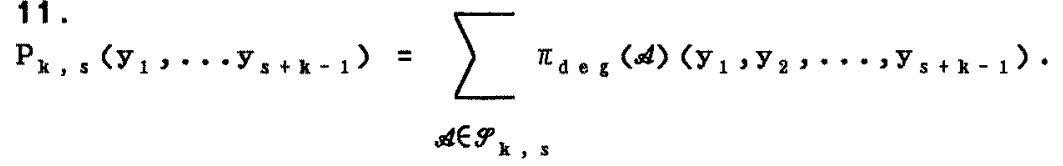

If $s=0$, the result is immediate. Otherwise, an induction shows that

$$
\begin{aligned}
& P_{k, s}\left(y_{1}, \ldots, y_{s+k-1}\right)=\sum_{i=1}^{k} y_{i} P_{k, s-1}\left(y_{2}, \ldots, y_{s+k-1}\right) \\
& =\sum_{i=1}^{k} \sum_{A^{\prime} \in \mathscr{S}_{k, s-1}} y_{i} \pi_{d e g}\left(A^{\prime}\right)\left(y_{2}, \ldots, y_{s+k-1}\right),
\end{aligned}
$$

Using the algorithm of construction of $\mathscr{S}_{\mathrm{k}, \mathrm{s}}$, each forest $A$ of $\mathscr{P}_{k, s}$ comes from a forest $\mathscr{A}^{\prime}$ of $\mathscr{S}_{k, s-1}$. If the vertex labeled 1 is a loop in $A$,

$$
\Pi_{d \in g}(A)\left(y_{1}, \ldots, y_{s+k-1}\right)=y_{1} \Pi_{d \in g}\left(A^{\prime}\right)\left(y_{2}, \ldots, y_{s+k-1}\right)
$$

Else, the vertex labeled 1 is a son of a vertex $i$ in [2..k] and

$$
\Pi_{d \in g}(A)\left(y_{1}, \ldots, y_{s+k-1}\right)=y_{i} \Pi_{d \in g}\left(A^{\prime}\right)\left(y_{2}, \ldots, y_{s+k-1}\right)
$$

Thus the lemma 11 is proved. We deduce

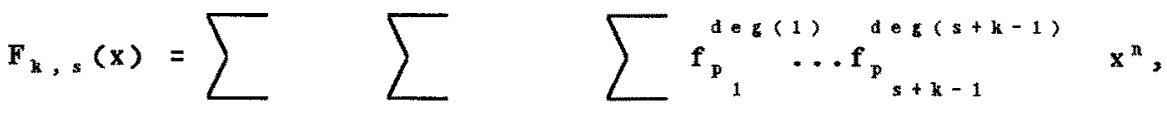

$$
\begin{aligned}
& \mathrm{n} \geqslant 1 \quad \mathrm{p}_{1}+\ldots+\mathrm{p}_{\mathrm{s}+\mathrm{k}-1}=\mathrm{n} \quad \mathscr{A} \in \mathscr{P}_{\mathrm{k}, s}
\end{aligned}
$$

and thus by factorisation, we obtain

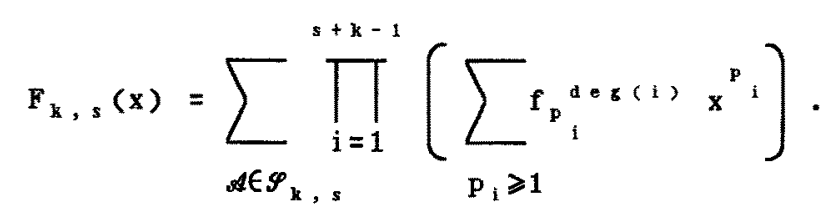

Consequently we have

$$
F_{k, s}(x)=\sum_{A \in \mathscr{P}_{k, s}} \Delta_{\mathrm{p}}(A)
$$


Using the notation $F_{k}(x)=\sum F_{\substack{k \\ s>0}}(x)$, we get

$$
\mathrm{F}_{\mathrm{k}}(\mathrm{x})=\sum_{\mathscr{A} \in \mathscr{P}_{\mathrm{k}}} \Delta_{\mathrm{p}}(A)
$$

and theorem 10 follows from property 7 .

\section{RELATIONS BETWEEN SUBSETS OF $g_{k}$}

In this section, we part the set $\mathscr{S}_{k}$ into particular subsets which basic properties allow us to decompose the formal power serie $\Phi\left(\mathscr{\varphi}_{k}\right)$ as sum of formal power series $P_{i} i_{2} \ldots i_{p}$ whose are given by

$$
P_{i i_{2}} \cdots i_{p}=\sum_{A \in E_{i} \ldots i_{p}} \varphi(A) .
$$

Then, we provide relations between these formal power series and we obtain two types of relations: - lemmas 12 to 15 will allow us to express $P_{1} \ldots . . . .$.
with respect to $P_{i} \ldots$ p $_{p+q}$

- lemma 16 will allows us to write $P_{i} \ldots i$ as $P_{j} \ldots j$
in order to apply lemmas 12 to $15 .{ }_{p+q}{ }_{1}{ }_{p+q}$

Most of these lemmas are based upon the relation of equivalence (definition 8) of particular types of forests and are deduced by the application of proposition 9 .

Notations: For every positive integer $p$, and for every p-uple in $[0 . . \mathrm{k}-1]^{\mathrm{p}}$, we denote by

- $E_{i} \cdots_{i}$ the set of forests belonging to $\varphi_{k}$ having at least $p+k-1$ vertices such that $\forall j \in[1 \ldots p], \delta(j)=i_{j}$, - e ${ }_{1} \ldots{ }^{i}$ the forest of $\varphi_{k}$ with $p+k-1$ vertices such that

$$
\forall j \in[1 \ldots p], \delta(j)=i_{j} .
$$

By convention, let E (resp. e) be $\mathscr{I}_{\mathrm{h}}\left(\operatorname{resp}, \mathscr{S}_{\mathrm{k}, 0}\right)$.

Example: the forest $A$ of the example 1 is exactly eq2oql and belongs to the sets $E, E_{2}, E_{22}, E_{220}, \ldots$ 
Lemma 12. For every p-uple $\left(\mathrm{i}_{1}, \mathrm{i}_{2}, \ldots, \mathrm{i}_{\mathrm{p}}\right)$ of $[0 \ldots \mathrm{k}-1]^{\mathrm{p}}$,

$$
E_{i} \ldots i_{p}=e_{i} \ldots i_{p}+\sum_{k=0}^{k-1} E_{i} \ldots i_{p}^{h},
$$

and

$$
P_{i} \ldots i=\varphi\left(e_{i}{ }_{i} \ldots i_{p}\right)+\sum_{i=0}^{k-1} P_{i} \ldots i_{p} \text {. }
$$

Indeed, a forest of $E_{1} \ldots i$ is

- either the forest $e_{i} \ldots i$,

- or a forest having at least p+k vertices, such that the half degree of the vertex $p+1$ is in $[0 \ldots k-1]$.

Examples: $E=e+\sum_{i=0}^{k-1} E_{i} \quad, \quad \Phi\left(\mathscr{g}_{k}\right)=y_{0}^{k-1}+\sum_{i=0}^{k-1} P_{i}$,

$$
E_{i}=e_{i}+\sum_{j=0}^{k-1} E_{i j}, \quad P_{i}=y_{1} y_{0}^{k-1}+\sum_{j=0}^{k-1} P_{i j} .
$$

Lemma 13. $P_{p(p-1) \ldots 10}=y_{0}^{p} y_{p+1} \Phi\left(\varphi_{k}\right)$

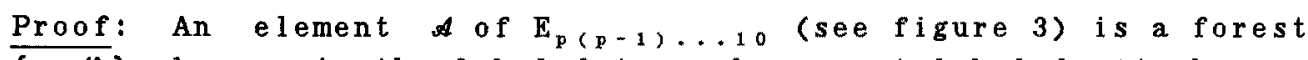
$\overline{\left\{u, A^{\prime}\right\}}$ where $u$ is the labeled tree whose root labeled p+1 has a loop and p sons labeled from 1 to $p$ and where is any forest of $\varphi_{k}$ where each label 1 of a vertex has been changed in $(1+p)$. Thus we have the announced result using the property 7 .

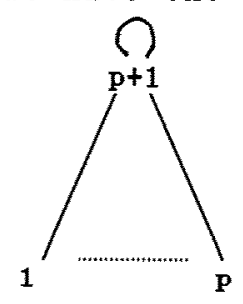

1
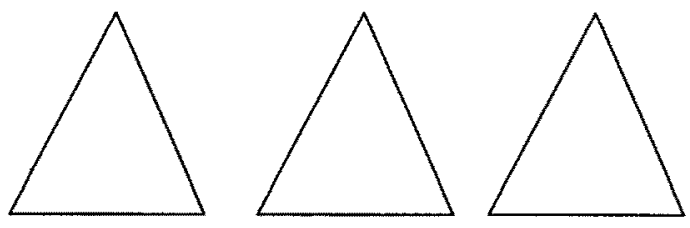

4

Figure 3. An element of $E_{p(p-1) \ldots 10}$ 
In the same way, we fix $j$ supplementary vertices and we get

Lemma 16. $P_{p(p-1) \ldots 10 i i_{1} \ldots j_{j}}=y_{0}{ }^{p} y_{p+1} P_{i} \ldots i_{j}$.

Example: $\mathrm{P}_{0}=\mathrm{y}_{1} \Phi\left(\mathscr{S}_{\mathrm{k}}\right), \mathrm{P}_{10}=\mathrm{y}_{0} \mathrm{y}_{2} \Phi\left(\mathscr{S}_{\mathrm{k}}\right), \mathrm{P}_{210}=\mathrm{y}_{0}{ }^{2} \mathrm{y}_{3} \Phi\left(\mathscr{S}_{\mathrm{k}}\right) \ldots$

and also $\mathrm{P}_{01}=\mathrm{y}_{1} \mathrm{P}_{1}, \mathrm{P}_{1 \mathrm{O}_{2} 3}=\mathrm{y}_{0} \mathrm{y}_{2} \mathrm{P}_{23} \ldots$

Lemma 15. Let $i_{1}=\alpha+p-1, i_{2}=\alpha+p-2, \ldots, i_{p}=\alpha$ and

then

$$
i_{p+1} \neq \alpha-1, i_{p+2} \neq \alpha-2, \ldots, i_{p+a} \neq 0
$$

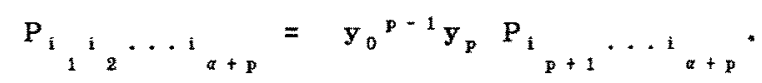

Let be a forest from $P_{i} i_{2} \ldots i_{\alpha+p}$ satisfying (6).

Then the vertex labeled $p+\alpha$ has exactly p sons labeled from 1 to $p$ as shown in figure 4. Indeed, the condition $i_{p+q} \neq \alpha-q$ in (6) means that the vertex labeled p+a has no son other than those labeled from 1 to $p$. Thus $A$ is equivalent to the forest obtained by displacing the vertices labeled from 1 to $p$ so that the vertex $p$ is a loop having $p-1$ sons labeled from 1 to $p-1$ as shown in figure 4. Therefore, we can use the lemma 14 which proves lemma 15 .

Example: $P_{3233}=y_{0} y_{2} P_{33}$.
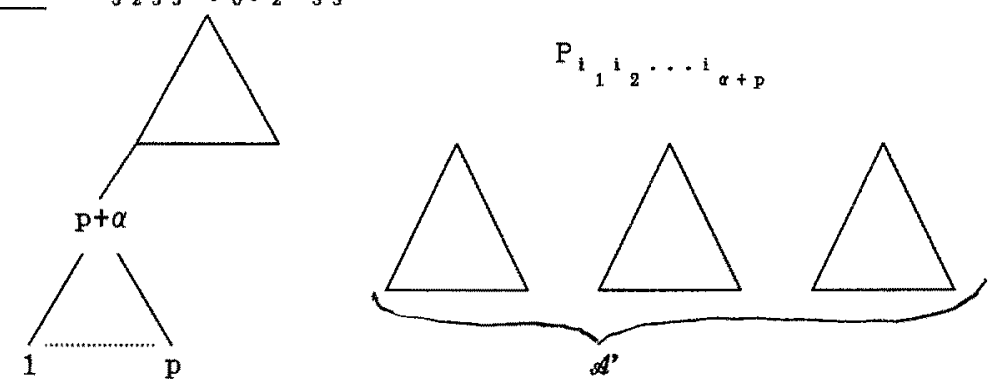

u
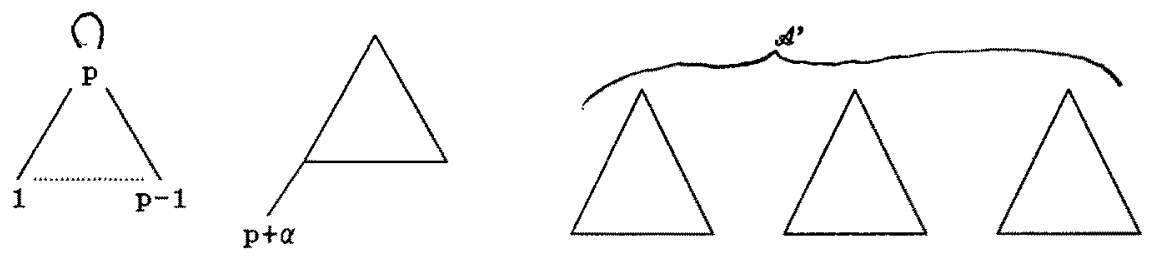

$\mathbf{y}_{0}{ }^{p-1} \mathbf{y}_{\mathbf{p}}$

$$
P_{i p+1} \cdots i_{\alpha+p}
$$

Figure 4. An example for lemma 16. 
Lemma 16. If the forest $A$ of $\mathscr{S}_{\mathrm{k}}$ is such that, for somme positive integers $\alpha, \beta, \gamma, \delta$, father $(\alpha)=\alpha+\beta+\gamma+\delta$ and father $(\alpha+\beta)=\alpha+\beta+\gamma$, then, A is equivalent to the forest obtained by the permutation of the subtrees raising from the vertices $\alpha$ and $\alpha+\beta$.

The proof is immediate because this transformation does not affect the entering degree. Thus we have the following

Corollary 17. If $i_{\alpha}=\beta+\gamma+\delta$ and $i_{\alpha+\beta}=\gamma$ then

$$
\begin{aligned}
& e_{i_{1}} \cdots_{\alpha} \cdots_{\alpha+\beta} \cdots_{p}=e_{i} \ldots{ }_{\alpha} \ldots_{\alpha+\beta} \cdots i_{p}, \\
& E_{i} \ldots i_{\alpha} \ldots i_{\alpha+\beta} \cdots i_{p}=E_{i} \ldots i_{\alpha}^{\prime} \cdots i_{\alpha+\beta}^{\prime} \cdots i_{p},
\end{aligned}
$$

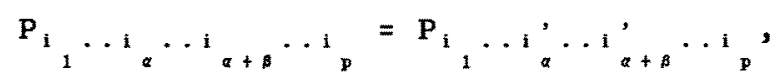

where i' $=\beta+\gamma$ and i' $\alpha+\beta=\gamma+\delta$.

Example: $\mathbf{P}_{20}=P_{11}$

\section{ENUMERATION OF THE K-SHAPED FORESTS}

In this section, we enumerate the k-shaped forests according to the distribution of each vertices in the forests.

The lemmas of the section 4 allow us to write out a linear system of equations satisfied by $\Phi\left(\mathscr{P}_{\mathrm{k}}\right)$. However, the number of equations increases exponentialy with k. Thus it was inefficient to write in Macsyma [12] an algorithm of generation for such a general system. Thus, we just solve explicitly the systems for $k=2,3$ and 4 .

Theorem 18. The generating function for $\mathrm{k}$-shaped forests are

$$
\begin{aligned}
& \text { for } k=2, \quad \Phi\left(\mathscr{S}_{2}\right)=\frac{\mathrm{y}_{0}}{\left(1-\mathrm{y}_{1}\right)^{2}-\mathrm{y}_{0} \mathrm{y}_{2}} \text {, } \\
& \text { for } k=3, \quad \Phi\left(\varphi_{3}\right)=\frac{y_{0}{ }^{2}}{\left(1-y_{1}\right)^{2}\left(1-y_{1}-y_{1}{ }^{2}\right)-2 y_{0} y_{2}\left(1-y_{1}{ }^{2}\right)-y_{0}{ }^{2}\left(y_{2}{ }^{2}+y_{3}\right)} \text {, } \\
& \text { and for } k=4, \Phi\left(\mathscr{S}_{4}\right)=\frac{\mathrm{y}_{0}{ }^{3}\left(1+\mathrm{y}_{1}{ }^{2}-\mathrm{y}_{0} \mathrm{y}_{2}\right)}{Q_{0}+Q_{1} \mathrm{y}_{0}+Q_{2} \mathrm{y}_{0}{ }^{2}+Q_{3} \mathrm{y}_{0}{ }^{3}+Q_{4} \mathrm{y}_{0}{ }^{4}} \text {, }
\end{aligned}
$$

$$
\text { where } \begin{aligned}
& Q_{0}=\left(1-\mathrm{Y}_{1}\right)^{2}\left(1-\mathrm{y}_{1}-\mathrm{y}_{1}{ }^{2}-\mathrm{y}_{1}{ }^{3}\right), \\
& Q_{1}=-2 \mathrm{Y}_{0} \mathrm{Y}_{2}\left(1-\mathrm{Y}_{1}\right)\left(2+\mathrm{Y}_{1}-\mathrm{Y}_{1}{ }^{2}-3 \mathrm{Y}_{1}{ }^{3}-3 \mathrm{Y}_{1}{ }^{4}-2 \mathrm{Y}_{1}{ }^{5}\right), \\
& Q_{2}=2 Y_{3}\left(Y_{1}-1\right)\left(1+3 Y_{1}+Y_{1}{ }^{2}+Y_{1}{ }^{3}\right)-Y_{2}{ }^{2}\left(1-4 Y_{1}{ }^{3}-6 Y_{1}{ }^{4}\right), \\
& Q_{3}=-\left(Y_{2}{ }^{3}+4 Y_{2} Y_{3}+Y_{4}\right)+2 Y_{1} Y_{2}\left(Y_{3}-Y_{2}{ }^{2}\right)-Y_{1}{ }^{2}\left(Y_{4}+4 Y_{2} Y_{3}+4 Y_{2}{ }^{3}\right), \\
& Q_{4}=\left(Y_{2}+Y_{3}\right)^{2}+Y_{2} Y_{4}-2 Y_{3}{ }^{2} .
\end{aligned}
$$


We write systems of linear equations by using first the lemma 12, then by applying lemmas 13 to 16 .

For $k=2$, we have

$$
\begin{aligned}
\Phi\left(\mathscr{\Phi}_{2}\right) & =\mathrm{Y}_{0}+\mathrm{P}_{0}+\mathrm{P}_{1}, \\
\mathrm{P}_{1} & =\mathrm{Y}_{0} \mathrm{Y}_{1}+\mathrm{P}_{10}+\mathrm{P}_{11}, \\
\mathrm{P}_{0} & =\mathrm{Y}_{1} \Phi\left(\mathscr{S}_{2}\right), \\
\mathrm{P}_{10} & =\mathrm{Y}_{0} \mathrm{Y}_{2} \Phi\left(\mathscr{S}_{2}\right), \\
\mathrm{P}_{11} & =\mathrm{Y}_{1} \mathrm{P}_{1} .
\end{aligned}
$$

Solving it, gives the result of theorem 18. We give in the annex the system for $k=3$ and 4 . They have been solved using the symbolic manipulation system MACSYMA from MIT [12]. We deduce from the theorem 19 the expression of $F_{k}(X)$ for $k=2,3$ and 4 using the theorem 11. Note that in the case $k=3$, we find the result of R. Stanley [10] that is

Proposition 19. Define

$$
N(n)=\sum\left(f_{n}+f_{n}+f_{n}\right)\left(f_{n_{2}}+f_{n_{3}}+f_{n_{4}}\right) \ldots\left(f_{n}+f_{n} f_{s+1}+f_{n}{ }_{s+2}\right) \text {, }
$$

where $\mathrm{f}$ is any function $\mathrm{f}: \mathbb{N} \rightarrow \mathbb{C}$ and where the sum is over all ordered partitions $\mathrm{n}_{1}+\mathrm{n}_{2}+\ldots+\mathrm{n}_{\mathrm{s}+2}=\mathrm{n}$ of $\mathrm{n}\left(\mathrm{n}_{1} \geqslant 1\right)$. By convention, a summand with $\mathrm{s}=0$ is 1 . Define

$$
F(X)=\sum_{n=1}^{\infty} N(n) X^{n}, \quad f=\sum_{n=1}^{\infty} f_{n} X^{n}, \quad A=X /(1-X) .
$$

Let * denote Hadamard product. Then

$$
\mathrm{A}^{2}
$$

$$
\left.F(X)=\frac{}{(1-f)^{2}\left(1-f-f^{2}\right)-2 A(f * f)\left(1-f^{2}\right)-A^{2}\left((f * f)^{2}+(f * f * f)\right.}\right)
$$

However, we did not find a general formula for $F_{k}(X)$, or, at least a recursive relation on these functions. Yet, the time of computation was very slight, and we think that it is possible to compute the result for $k=5$.

\section{REFERENCES}

[1] C. BERGE, Graphes et hypergraphes, Dunod, Paris (1970).

[2] N.G. de BRUIJN and B.J.M. MORSELT, A note on plane trees, J. Comb. Th. 2 (1967), 27-34.

[3] R. CORI and B. VAUQUELIN, Planar maps are well labeled trees, Canadian J. of Math. 33 (1981) 1023-1042.

[4] M.P. DELEST, Utilisation des langages algébriques et du calcul formel pour le codage et l'énumération des polyominos, Thèse d'Etat, Université de Bordeaux I, 1987.

[5] J.M. FEDOU, Enumération de certains polyominos selon les parametres périmètre et aire, mémoire de D.E.A, Bordeaux I, 1987 . 
[6] P. FLAJOLET, Analyse d'algorithmes de manipulation d'arbres et de fichiers, Cahiers du B.U.R.0. 34-35(1981), 1-209.

[7] A. JOYAL, Une théorie combinatoire des séries formelles, Adv. in Math., 42 (1981), 1-82.

[8] D. KNUTH, The art of computer programming, Vo1. 1, Addison Wesley Reading, 1968 .

[9] J.W. MOON, A note on an identity and labelled forests, Caribb. J. Math., 3, (2) 59-65.

[10] R.P. STANLEY, Generating function, Studies in Comb, 100-148, 17, Math. Assoc. America Washington DC 78 .

[11] J.M. STEYEART, Complexité et structure des algorithmes, Thèse Université Paris VII, 1984.

[12] SYMBOLICS INC., Macsyma Reference manual version ten, third printing, december 1984.

[13] G. VIENNOT, Heap of pieces: Basic definitions and combinatorial lemmas, in Combinatoire Enumérative, UQAM 1985, Montréal, G. Labelle et P. Leroux ed., Lecture Notes in Mathematics, ${ }^{\circ} 1234$, Springer Verlag, 321-350, 1986 .

[14] G. VIENNOT, Trees, Rivers, RNAs and many other things, preprint, Bordeaux I, 1987.

\section{ANNEXE}

For $\mathbf{k}=3$,

$$
\begin{aligned}
\Phi\left(\mathscr{P}_{3}\right) & =y_{0}{ }^{2}+P_{0}+P_{1}+P_{2}, \\
P_{1} & =y_{0} y_{1}+P_{10}+P_{11}+P_{12}, \\
P_{2} & =y_{0}{ }^{2} y_{0}+P_{20}+P_{21}+P_{22}, \\
P_{20} & =y_{0}{ }^{2} y_{1}+P_{200}+P_{201}+P_{202}, \\
P_{21} & =y_{0} y_{2}+P_{210}+P_{211}+P_{212}, \\
P_{22} & =y_{0} y_{1}+P_{220}+P_{221}+P_{222}, \\
P_{0} & =y_{1} \Phi\left(\mathscr{P}_{3}\right), \\
P_{10} & =y_{0} y_{2} \Phi\left(\mathscr{P}_{3}\right), \\
P_{11} & =y_{0} P_{1}, \\
P_{12} & =y_{0} P_{2}, \\
P_{200} & =P_{110}=y_{1} P_{10}, \\
P_{201} & =P_{111}=y_{1} P_{11}, \\
P_{202} & =P_{112}=y_{1} P_{12}, \\
P_{210} & =y_{0} y_{3} \Phi\left(\mathscr{P}_{3}\right), \\
P_{211} & =y_{0} y_{2} P_{1} .
\end{aligned}
$$

For $k=4$,

$$
\begin{aligned}
\Phi\left(\mathscr{S}_{4}\right) & =\mathrm{y}_{0}{ }^{3}+\mathrm{P}_{0}+\mathrm{P}_{1}+\mathrm{P}_{2}+\mathrm{P}_{3}, \\
\mathrm{P}_{0} & =\mathrm{y}_{1} \Phi\left(\mathscr{S}_{4}\right), \\
\mathrm{P}_{1} & =\mathrm{y}_{0}{ }^{3} \mathrm{y}_{1}+\mathrm{P}_{10}+\mathrm{P}_{11}+\mathrm{P}_{12}+\mathrm{P}_{13}, \\
\mathrm{P}_{2} & =\mathrm{y}_{0}^{3} \mathrm{y}_{0}+\mathrm{P}_{20}+\mathrm{P}_{21}+\mathrm{P}_{22}+\mathrm{P}_{23}, \\
\mathrm{P}_{3} & =\mathrm{y}_{0}{ }^{3} \mathrm{y}_{0}+\mathrm{P}_{30}+\mathrm{P}_{31}+\mathrm{P}_{32}+\mathrm{P}_{33}, \\
\mathrm{P}_{10} & =\mathrm{y}_{0} \mathrm{y}_{2} \Phi\left(\mathscr{S}_{4}\right), \\
\mathrm{P}_{11} & =\mathrm{y}_{1} \mathrm{P}_{1},
\end{aligned}
$$




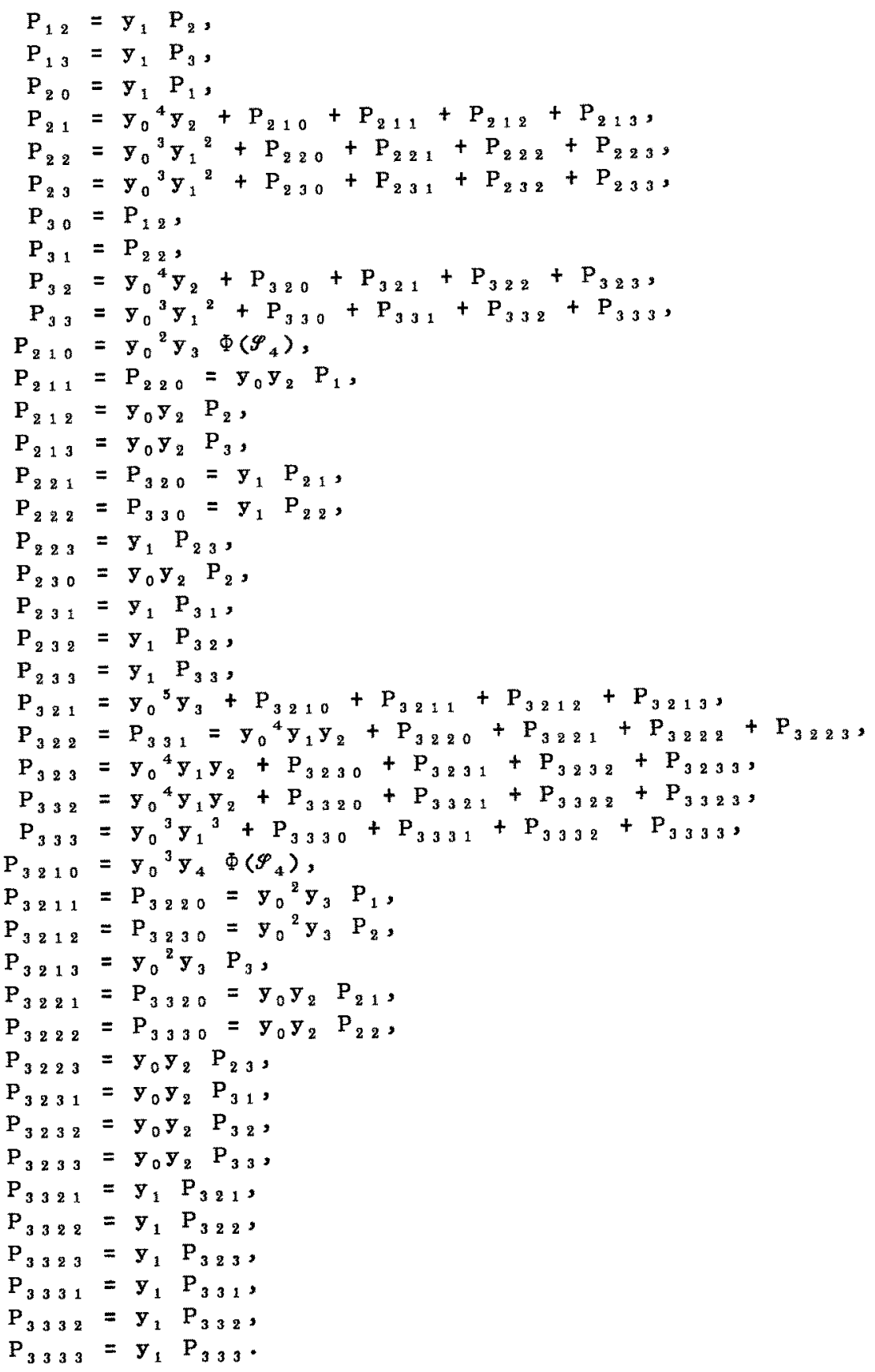

